\title{
Peripheral Repetitive Magnetic Stimulation: A Novel Approach for Hand Rehabilitation in Carpal Tunnel Syndrome - A Pilot Study
}

\author{
Savulescu Simona Elena, Grozoiu Lavinia, Popa Florina, Dumitru Luminita, and Berteanu Mihai
}

\begin{abstract}
Carpal tunnel syndrome is one of the disabling conditions that affects human participation and quality of life. The surgical treatment of diagnosed carpal tunnel syndrome is well known to be more effective than the conservative methods, but this pilot study on five subjects diagnosed with carpal tunnel syndrome gives hope that repetitive magnetic stimulation applied to the wrist could be effective in the rehabilitation of the hand neuro-muscular functionality and symptomatology. The goal of this study was to evaluate the short and medium effects of peripheral repetitive magnetic stimulation (RMS) on carpal tunnel pain and hand's loss of function. Two weeks of daily sessions treatment, each lasting 10 minutes of stimulation, have shown significant improvement of the functional score (Boston Questionare), hand grip force and also of symptomatic status (Boston Questionare, PainDetect Questionaire).
\end{abstract}

Index Terms-Peripheral repetitive magnetic stimulation, carpal tunnel syndrome, rehabilitation, hand grip.

\section{INTRODUCTION}

Carpal tunnel syndrome is one of the disabling conditions that affect human participation and quality of life. It is the most common of all entrapment neuropathies [1] and it means that the median nerve is compresed within the carpal tunnel. The median nerve is a mixt nerve so we have senzitive and motor simptoms. Depending on the degree of the compresion, the lesion of the nerve can be minor, consisting in demielination, or it can be more severe when axonal implication ocurs. The patient presents with numbness in the first 3 fingers, pain located at the wrist tipically at night and sometimes reports the loss of hand grip force.

The diagnosis of carpal tunnel syndrome can be a clinical one, when patient relates the specific simptoms and have Tinnel or Fallot signs positive, but most relevant are the

Manuscript received April 21, 2015; revised June 5, 2015. This work was supported by the Sectoral Operational Programme Human Resources Dvelopment (SOP HRD), financed from the European Social Fund and by the Romanian Government under the contract number POSDRU/159/1.5/S/132395.

Simona Elena Savulescu is with the Rehabilitation Medicine Department of Elias University Hospital, 17 Marasti Bd, Bucharest, Romania (PC 011461) (e-mail: simona.manoila@yahoo.com).

Lavinia Grozoiu is with the Rehabilitation Medicine Department of Elias University Hospital, Bucharest, Romania (PC 011461) (e-mail: laviniagrozoiu@gmail.com).

Florina Popa is with University of Medicine, Lucian Blaga, Sibiu, Romania.

Dumitru Luminita and Berteanu Mihai are with Carol Davila University of Medicine and Pharmacy, Bucharest, Romania (e-mail: lumivd@yahoo.com, mberteanu@gmail.com). electrophisiological findings. An EMG electromyography) and NCS (nerve conduction study) must be done to every patient in order to exclude a radiculopathy or a double crush syndrome. The specific NCS findings in carpal tunnel syndrome are: prolonged distal latencies, small Snap (sensitive nerve action potential) and Cmap (compound muscle action potential) amplitudes or decreased senzitive or motor conduction velocities. At the needle detection examination, if there is a axonal loss, the spontanous activity consisting in PSW(Positive Short Waves) and fibrilation can be found, and also neurogenic MUAP (motor unit action potential). At maximum contraction the pattern of recrutation

is simple or intermediar [2]. Conservative treatment may include corticoid injection, physical therapy (kinetotherapy, ultrasound, laser), bracing and alternative therapies. While surgery is known to be the definitive treatment for CTS, some people symptoms improve spontanously and this may relate to activity daily living modification [1]. Many publications about CTS surgical treatment report excellent results and low complication rates [3]-[5], while others report many kind of complications, such as the recurrence of the carpal tunnel syndrome [6]-[8]. Peripheral nerve repetitive magnetic stimulation might be a new physical agent than can be used to relieve pain and to recover hand function. It is an easy to use comfortable method for the pacient in order to obtain nerve stimulation. Based on the principles enunciated in the Gate Control Hypothesis that was published in 1968, stimulation-produced analgesia (SPA) has been a subject to intensive laboratory and clinical investigation [8]. Repetitive magnetic stimulation at the periphery (PRMS), i.e. over spinal roots, nerves or muscles, represents a new painless and noninvasive approach that can contribute to motor recovery [10].

The main objectives in hand neurorehabilitaion is to regain sensibility and muscle force, to relieve pain in order to restore hand grip and function. RMS is lately known to have positive therapeutic effects on myofascial pain both short and medium-term [11]. It's also known that a single session of RMS resulted in significant improvement of pain associated with lumbar spondylosis in a randomized, double-blind, placebo-controlled setting [12].

\section{OBJECTIVE}

The Objective of this pilot study is to determine if peripheral repetitive magnetic stimulation located at the wrist has an impact on nerve regeneration and clinical improvement of hand symptomatology and functionality. 
The study is designed to see if there are some perspectives for the treatment with high power magnetic stimulator and a futher study is designed to compare the result with those from a control group that will be treated with a Sham coil, but also with the results from a group treated with steroid injection.

\section{MATERIAL}

The pilot study was done on a group of 5 patients with a clinical reference of carpal tunnel syndrome, one of them with the right hand affected and the rest of them with the left hand affected.

In order to obtain a good diagnostic we also used Boston Questionaire, a hand grip dynamometer and an electromyography. For the treatment we used a MagVenture MagPro X100 stimulator with a RT-120 racetrack coil. Boston Questionaire is designed to to be applied in patients with carpal tunnel syndrome, with the purpose of evaluating the severity of symptoms (Severity of symptoms-SS) and the degree of manual skill (Functional Status-FS) [13]. Electromyography: We Used a Nicolet Compact Meridian. The NCS were done with surface electrodes and the detection EMG was done using a $50 \mathrm{~mm} \times 26 \mathrm{~g}$ needle. The Dynamometer we used is KERN MAP Version 11. 06/2010 Pain Detect Questionaire is known to evaluate the neuropatic pain and contains questions about the subjective perception of pain, but also objective clinical findings.

\section{METHOD}

The group of 5 patients were clinically diagnosed with carpal tunnel syndrome and they were refered to an EMG test. After having the consent of information signed, they were recruted to the study (T1), they have been EMG tested,the hand grip force was testet with the dynamometer (also the healthy hand) and Boston Questionare, PainDetect Questionare were administrated. Each of them had 10 sessions of RMS with the coil located at the wrist. The magnetic stimulation protocol consisted in 100 trains of 5 pulses/train, delivered at $10 \mathrm{~Hz}$, at an interval of 5 seconds between trains. At the begining and at the end of every session the grip force was tested using the hand grip dynamometer.

The protocol of EMG test included NCS on median nerve consisting in: the latencies of Snap and Cmap, the amplitude of Snap and Cmap, SNCV (Sensitive Nerve Conduction Velocity) and the neadle detection examining the spontanous activity registred from APB(Abductor Policis Brevis) muscle, MUAP analisys and the pattern of interference at the maximum contraction. We consent that if there was no spontanous activity it will be noted as „0" and if it's present it will be noted as "1". Likewise it was consented that if the morphology of MUAP is normal it will be noted as "0", between 3-4 phases, crenelation or satelite potentials $=1,5-6$ pases $=2$ and more than 7 phases $=3$.

Maximum force contraction is noted 3 if it is an interference pattern, 2 if it's intermediar and 1 if it is a simple one.

At the end of the 10 sessions (T10) they also have been
EMG tested, hand grip force tested and the two questionaires have been administrated.

Even if the Boston Questionare is a self administrated test, we wanted to personaly question the patients in order to eliminate the subjective errors.

\section{RESULTS}

\section{A. The EMG Findings}

A significant improvement of SNCV was observed at only one patient (from 28 to $38 \mathrm{~m} / \mathrm{s}$ ), two of them had a minor improve of SNCV From 32, respectively 31 to 35, respectively $36 \mathrm{~m} / \mathrm{s}$ ) and two patient had no modification of the sensitive conducion velocity. No major good results in Cmap latencies was detected (improvements of $0.3 \mathrm{~m} / \mathrm{s}$ ) and the prolongued Cmap latencie registred from one patient can be due to an error of distance mesurement from the point of stimulation to the registration electrode.

No significant impovement of Cmap or Snap amplitude was observed after the RMS of median nerve at the wrist meaning that the number of healthy axons of injured median nerve is not recovering (growing). The results show no influence of RMS on SPA recorded from APB muscle except one case of remiting SPA.

Concerning the MUAP morphology, in 3 cases it was observed the new poliphasic morphology of MUAP, one case showed an increased number of phases and one showed no impoved poliphasy. Maximal contraction pattern was improved in two cases and the rest showed the same level (see Table I-Table II).

TABLE I: THE VARIATION OF THE SNCV AND CMAP LATENCIES BEFORE AND AFTER THE TREATMENT

\begin{tabular}{|c|l|l|l|l|}
\hline Patient & $\begin{array}{l}\text { SNCV } \\
(\mathrm{m} / \mathrm{s}) \\
\mathrm{T} 1\end{array}$ & $\begin{array}{l}\text { SNCV } \\
(\mathrm{m} / \mathrm{s}) \\
\mathrm{T} 10\end{array}$ & $\begin{array}{l}\text { Cmap Latency(ms) } \\
\text { T1 }\end{array}$ & $\begin{array}{c}\text { Cmap } \\
\text { Latency(ms) } \\
\text { T10 }\end{array}$ \\
\hline 1 & 31 & 31 & 5.4 & 5 \\
\hline 2 & 30 & 30 & 7.2 & 7.7 \\
\hline 3 & 28 & 38 & 7.3 & 7.2 \\
\hline 4 & 32 & 35 & 4.8 & 4.8 \\
\hline 5 & 31 & 36 & 5 & 4.4 \\
\hline
\end{tabular}

TABLE II: THE VARIATION OF THE AMPLITUDINE OF SNAP AND CMAP

\begin{tabular}{|r|l|c|c|c|}
\hline \multirow{5}{*}{ Patient } & $\begin{array}{l}\text { Snap } \\
\text { Amplitude } \\
(\mu \mathrm{V})-\mathrm{T} 1\end{array}$ & $\begin{array}{l}\text { Snap } \\
\text { Amplitude } \\
(\mu \mathrm{V})-\mathrm{T} 10\end{array}$ & $\begin{array}{l}\text { Cmap } \\
\text { Amplitude } \\
(\mathrm{mV})-\mathrm{T} 1\end{array}$ & $\begin{array}{l}\text { Cmap } \\
\text { Amplitude } \\
(\mathrm{mV})-\mathrm{T} 10\end{array}$ \\
\hline 1 & 10.5 & 12.6 & 6.16 & 5.8 \\
\hline 2 & 1.06 & 1 & 9.9 & 8.7 \\
\hline 3 & 12.9 & 12.1 & 7.3 & 8.6 \\
\hline 4 & 4.17 & 4.2 & 0.28 & 0.3 \\
\hline 5 & 5.57 & 5.88 & 3.88 & 3.92 \\
\hline
\end{tabular}

The results show no influence of RMS on SPA recorded from APB muscle except one case of remiting SPA. Concernic the MUAP morphology in 3 cases it was observed the new poliphasic morphology of MUAP, one case show an increased number of phases and one sho no impoved poliphasy. Maximal contraction pattern was improved in two cases and the rest show the same level. 


\section{B. The Hand Grip Force Results}

All the patients had a significant improvement in hand grip force at the end of all ten RMS sessions (with $6 \mathrm{Kg}$, in average). The last patient had a complete recovery of hand grip force (compared with the healty hand) (see Table III).

TABLE III: THE HAND GRIP FORCE VARIATION BEFORE AND AFTER THE TREATMENT

\begin{tabular}{|c|c|c|}
\hline Patient & $\begin{array}{c}\text { Hand } \\
\text { Grip Force }(\mathrm{Kg}) \\
\mathrm{T} 1\end{array}$ & $\begin{array}{c}\text { Hand } \\
\text { Grip Force }(\mathrm{Kg}) \\
\text { T10 }\end{array}$ \\
\hline 1 & 21.3 & 25.1 \\
\hline 2 & 14.9 & 23.9 \\
\hline 3 & 15 & 23.4 \\
\hline 4 & 10.5 & 19.5 \\
\hline 5 & 32 & 39.1 \\
\hline
\end{tabular}

\section{The Boston Questionaire Results}

The normal value for the SS and FS score in a healthy patient is 1. All the patients had a better SS and FS score and furher studies with a larger number of patients must be done to determine if there is statistic semnificative improvement of symptoms and functional status (see Table IV).

TABLE IV: THE VARIATION OF BOSTON QUESTIONAIRE RESULTS (SS= SEVERITY OF SYMPTOMS, FS = FUNCTIONAL STATUS) BEFORE AND AFTER THE TREATMENT.

\begin{tabular}{|r|l|l|l|l|}
\hline Patient & SS T1 & SS T10 & FS T1 & FS T10 \\
\hline 1 & 3.18 & 1.81 & 3 & 2.14 \\
\hline 2 & 3.09 & 1.54 & 3.14 & 2.14 \\
\hline 3 & 3 & 2.54 & 2.75 & 2 \\
\hline 4 & 3.1 & 2.2 & 3.2 & 2 \\
\hline 5 & 2.45 & 1.81 & 2.14 & 1.28 \\
\hline
\end{tabular}

\section{The Pain Detect Questionaire Results}

All the Patients registred a dropp of the score meaning that the pain and numbness were impoved but did not disappear (variations of 5 points in average) (see Table V).

TABLE V: THE VARIATION OF PAIN DETECT QUESTIONAIRE RESULTS BEFORE AND AFTER THE TREATMENT

\begin{tabular}{|l|l|l|}
\multicolumn{3}{|c|}{ BEFORE AND AFTER THE TREATMENT } \\
\hline Patient & Score T1 & Score T10 \\
\hline 1 & 16 & 10 \\
\hline 2 & 16 & 11 \\
\hline 3 & 6 & 4 \\
\hline 4 & 9 & 7 \\
\hline 5 & 12 & 6 \\
\hline
\end{tabular}

\section{DISCUSSION}

The EMG findings were not very encouraging. Some minor improvements of NCS of median nerve at the carpal tunel level were observed, especially concerning the sensitive nerve conduction velocity with no significant Snap or Cmap increased amplitude but it was observed an enhanced poliphasism at the end of the 10 sessions concurrent with a richer pattern of recrutation at some patients when developing maximum force contraction.

The 5'th patient had the best results at EMG studies, questionare scores and hand grip force after the treatment showing maybe a better result in acute stages of carpal tunnel syndome(the patient had acute denervation and minor hand grip force loss at the begining of the treatment).
It is not known if the poliphasic MUAP is due to the regeneration of the nerve or if it's consecutive to reconstruction of motor unit because of collateral sprouting. More feasible is the second theory in accordance with the enhanced muscule force and with a better interference patern .

A better result was determined concerning the Boston questionaire score at the end of the treatment. All the pacients had a drop of the symptome score and functional score meaning the improvement of the sensation and function of the hand.

Consisstent with BQ result (SS-score) was also the PainDetect Questionaire score, which means that RMS has a fast, good effect on patient's symptoms ( pain and numbness).

An enhanced hand grip force and function of the hand derives from the drop of functional score and the better force value mesured with the dynamometer at the end of the 10 sessions.

\section{CONCLUSION}

These preliminar results show that median nerve repetive magnetic stimulation could be a helpful tool in the neurorehabilitation of carpal tunnel syndrome and a further study is designed to compare the effects of RMS in neurorehabilitation with sham and with the effects of steroid injection.

\section{ACKNOWLEDGMENT}

This paper is supported by the Sectoral Operational Programme Human Resources Development (SOP HRD), financed from the European Social Fund and by the Romanian Government under the contract number POSDRU/159/1.5/S/132395.

\section{REFERENCES}

[1] C. Hans et al., "Current options for nonsurgical management of carpal tunnel syndrome," International Journal of Clinical Rheumatology, vol. 5, no. 1, 2010, pp. 129-142.

[2] M. Berteanu, "Biofeedback-ul electromiografic. Baze neurofiziopatologice si aplicatii in recuperare medicala," Edition Universitara, Carol Davila, 2006, pp. 81-84.

[3] T. K. Cobb, B. K. Dalley, R. H. Posterapo, and R. C. Lewis, "Anatomy of the flexor retinaculum," Journal Hand Surg Am, vol. 18, pp. 91-99, 1993.

[4] G. S. Phalen, "The carpal tunnel syndrome," Journal Bone Joint Surg Am, vol. 48, pp. 211-228, 1966.

[5] G. E. Omer, "Median nerve compreesion at the wrist," Hand Clin, vol. 8, pp. 317-324, 1992.

[6] H. Seradge and E. Seradge, "Piso-triquetral paqin syndrome after carpal tunnel release," Journal Hand Surg Am, vol. 14, pp. 858-862, 1989.

[7] A. V. Zumiotti, P. E. Ohno, F. S. Prada, and R. J. Azze, "Complicações do tratamento cirúrgicoda síndrome do túnel do carpo," Rev. Bras Ortop, vol. 31, pp. 199-202, 1996.

[8] W. Kluge, R. G. Simpson, and A. C. Nicol, "Late complications after open carpal tunnel decompressiom," Journal Hand Surg Br, vol. 21, pp. 205-207, 1996.

[9] M. S. Hicks and J. Salamon, "Stimulation of the central and peripheral nervous system for the control of pain," Journal of Clinical Neurophysiology, vol. 14, no. 1, pp. 46-62, 1997.

[10] L. D. Beaulieu and C. Schneider, "Effects of repetitive peripheral magnetic stimulation on normal or impaired motor control; A review," Neurophysiologie Clinique/Clinical Neurophysiology, vol. 43, no. 4, pp. 251-256, 2013 
[11] S. Nicola et al., "Therapeutic effects of peripheral repetitive magnetic stimulation on myofascial pain syndrome," Clinical Neurophysiology, pp. 350-358, 2003.

[12] L. L. Yew et al., "A randomized, placebo-controlled Trial of repetitive spinal magnetic stimulation in lumbosacral spondylotic pain," Pain Medicine, vol. 12, no. 7, 2011.

[13] D. W. Levine, B. P. Simmons, M. J. Koris, L. H. Daltroy, G. G. Hohl, A H. Fossel et al., "A self administered questionnaire for the assessment of severity of symtoms and functional status in carpal tunnel syndrome," Journal Bone Joint Surg Am, vol. 75, pp. 1585-1592, 1993.

Simona Savulescu was born in Tirgu-Jiu in 1981. She obtained her bachelor degree in general medicine from U.M.F Carol Davila Bucharest, Romania, in 2007 then made the residency until 2012 in the Department of Rehabilitation Medicine of Elias University Hospital and since 2011 is a Ph.D student in Medical Science at U.M.F Carol Davila Bucharest, Romania.

She is a consultant physicia in the Rehabilitation Medicine Department of Elias Univeristy Hospital, Bucharest, Romania since 2012. She is also the electro physiologist of the Department with a high interest in magnetic field therapy.

Dr. Simona Savulescu is an active member of Romanian Society of Rehabilitation, International Society of Physical and Rehabilitation Medicine and of Romanian Society of Electro Diagnostic Neurophysiology.

Lavinia Grozoiu was born in Moreni, Romania in 1984. She obtain her bachelor degree in general medicine from the Transilvania University of Medicine, Brasov, Romania, in 2008. After her residency training in Bucharest, she conducted an 8 month internship in Berlin, Germany.

She is currently pursuing her study for $\mathrm{PhD}$ degree in medical rehabilitation, at Carol Davila University of Medicine and Pharmacy Bucharest in collaboration with Medical Park Berlin Humboldtmühle, Charite-University Medicine Berlin. Her research interests include stroke rehabilitation and repetitive magnetic stimulation.

\section{Dr. Lavinia Grozoiu is a member of Romanian Society of Rehabilitation}

Luminita Dumitru was born in Draganesti Olt on December 30, 1968. He obtained her bachelor degree in general medicine in 1993 from U.M.F Carol Davila Bucharest, Romania, became in 2002 a consultant physician at Colentina Hospital, Bucharest, in 2004 a second consultant at Elias University. Since 2006 she is an assistant professor at U.M.F Carol Davila Bucharest, Romania and a Ph.D from 2014 in the Rehabilitaion Medicine Field .

She is a second consultant physician since 2004 at Elias University Hospital, Bucharest, Romania

One of the major field of her research interest is the electrotheraphy and its effects on heart rhythm disorders.

Asst. Prof. Dr. Luminita Dumitru is an active member of Romanian Society of Rehabilitation, International Society of Physical and Rehabilitation Medicine.

Mihai Berteanu was born in Bucharest, Romania in 1962. He obtained his bachelor degree in general medicine from the Carol Davila University of Medicine and Pharmacy, Bucharest, in 1987. After his residency training in Romania, he performed multiple internships abroad (Bruxelles 1997, Berlin 1998, Washington 2000, Winterthur 2004, Innsbruck 2006). His completed his $\mathrm{PhD}$ degree in field of rehabilitation using electromyography biofeedback, in 2013. He is now a professor of Rehabilitation Medicine at the Carol Davila University.

He is now the head of The Rehabilitation Medicine Department from Elias University Hospital Bucarest, Romania. He has numerous publications in international journals and became a professor of Rehabilitation at the Carol Davila University.

Prof. Dr. Mihai Berteanu is a member of the European Board and AAPM\&R since 2005. He is also a member of World Federation of Neurological Rehabilitation, ISPRM, GESET. 